Supplement of Clim. Past, 15, 1443-1461, 2019

https://doi.org/10.5194/cp-15-1443-2019-supplement

(c) Author(s) 2019. This work is distributed under

the Creative Commons Attribution 4.0 License.

(c) (1)

Supplement of

\title{
Past climate and continentality inferred from ice wedges at Batagay megaslump in the Northern Hemisphere's most continental region, Yana Highlands, interior Yakutia
}

Thomas Opel et al.

Correspondence to: Thomas Opel (thomas.opel@awi.de, t.opel@sussex.ac.uk)

The copyright of individual parts of the supplement might differ from the CC BY 4.0 License. 
Table S1. Studied ice wedges.

\begin{tabular}{|c|c|}
\hline Photograph & Ice-wedge features \\
\hline \multicolumn{2}{|c|}{$\begin{array}{l}\text { Ice wedge B17-IW1 } \\
\text { Batagay megaslump - Lower Ice Complex }\end{array}$} \\
\hline & $\begin{array}{l}\text { Width: } \sim 0,5 \mathrm{~m} \\
\text { Visible vertical extension: } \sim 1 \mathrm{~m} \\
\text { Colour: dirty grey } \\
\text { Ice veins: partly detectable, width up to } 1 \mathrm{~cm} \\
\text { Sediment content: very little to little } \\
\text { Organic content: very little } \\
\text { Bubble content: very high; very small bubbles }(<0.5 \mathrm{~mm}) \text {; } \\
\text { often rather cloudy; following the ice veins } \\
\text { Remarks: truncated; upper left part partly degraded } \rightarrow \\
\text { shoulder + pool ice }\end{array}$ \\
\hline
\end{tabular}




\begin{tabular}{|c|c|}
\hline \multicolumn{2}{|c|}{$\begin{array}{l}\text { Ice wedge B17-IW6 } \\
\text { Batagay megaslump - Upper Ice Complex }\end{array}$} \\
\hline & $\begin{array}{l}\text { Width: } \sim 0.5 \mathrm{~m} \\
\text { Visible vertical extension: } \sim 1.5 \mathrm{~m} \\
\text { Colour: grey } \\
\text { Ice veins: clearly detectable, up to } 1 \mathrm{~cm} \text { wide } \\
\text { Sediment content: high (sandy ice wedge) } \\
\text { Organic content: very little } \\
\text { Bubble content: very little to medium; some spherical } \\
\text { bubbles up to } 2 \mathrm{~mm} \text { in diameter; some elongated bubbles up } \\
\text { to } 2 \mathrm{~cm} \text { long and } 1-2 \mathrm{~mm} \text { in diameter; very small bubbles in } \\
\text { centre of veins }\end{array}$ \\
\hline \multicolumn{2}{|c|}{$\begin{array}{l}\text { Ice wedge B17-IW5 } \\
\text { Batagay megaslump - Upper Ice Complex }\end{array}$} \\
\hline & $\begin{array}{l}\text { Width: } ~ 1.6 \mathrm{~m} \\
\text { Visible vertical extension: }>5 \mathrm{~m} \\
\text { Colour: clear, grey ice } \\
\text { Ice veins: clearly detectable, partly with sediment inclusions, } \\
\text { about } 1 \mathrm{~cm} \text { wide } \\
\text { Sediment content: little to medium } \\
\text { Organic content: very little } \\
\text { Bubble content: medium to high; spherical bubbles } 2-3 \mathrm{~mm} \\
\text { in diameter, elongated bubbles up to } 1 \mathrm{~cm} \text { long and } 1-2 \mathrm{~mm} \\
\text { in diameter; very small cloud-like bubbles in centre of veins } \\
\text { Remarks: none }\end{array}$ \\
\hline
\end{tabular}




\begin{tabular}{|l|l|}
$\begin{array}{l}\text { Composite wedges B17-IW2 (left) and B17-IW3 (right) } \\
\text { Batagay megaslump - Upper Sand unit }\end{array}$ & $\begin{array}{l}\text { Width: } 0.2-0.3 \mathrm{~m} \text { (left) and } 0.2 \mathrm{~m} \text { (right), respectively } \\
\text { Visible vertical extension: } \sim 3 \mathrm{~m} \\
\text { Colour: grey } \\
\text { Ice veins: partly detectable; up to 0.5-1 cm wide, very thin } \\
\text { sand veins } \\
\text { Sediment content: high to very high (composite wedges) } \\
\text { Organic content: not detectable } \\
\text { Bubble content: very little; very small bubbles } \\
\text { Remarks: truncated; both wedges unite downwards into one } \\
\text { wedge. }\end{array}$ \\
\hline
\end{tabular}




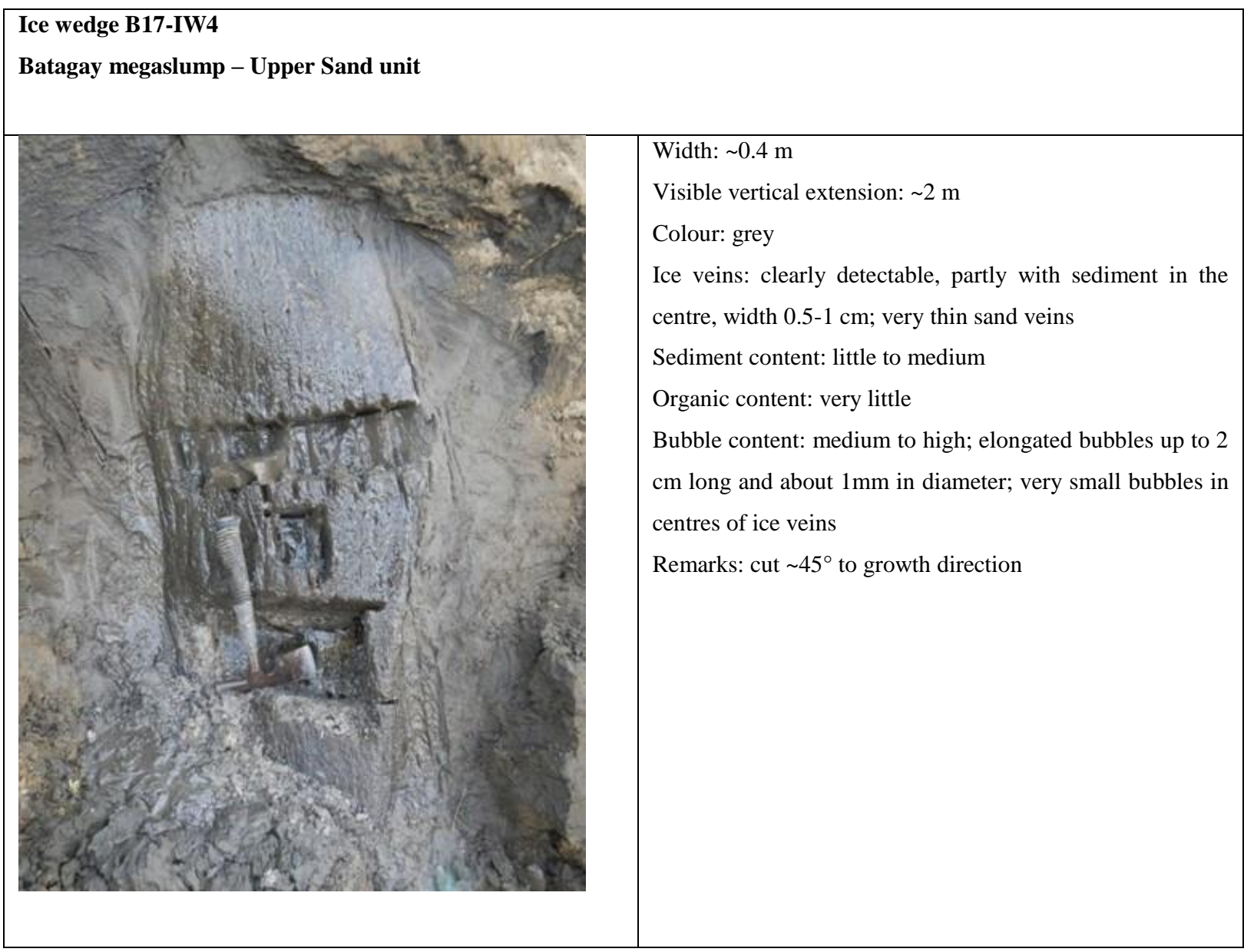




\begin{tabular}{|l|l|}
\hline $\begin{array}{l}\text { Ice wedge A17-IW3 } \\
\text { Adycha river - Late Holocene river bank }\end{array}$ & $\begin{array}{l}\text { Width: } \sim 0.3 \mathrm{~m} \\
\text { Visible vertical extension: } \sim 3 \mathrm{~m} \\
\text { Colour: milky white } \\
\text { Ice veins: clearly detectable, up to } 1 \mathrm{~cm} \text { wide } \\
\text { Sediment content: little } \\
\text { Organic content: little } \\
\text { Bubble content: very high; some spherical bubbles up to } 1 \\
\text { mm in diameter; many small, partly elongated bubbles along } \\
\text { ice veins } \\
\text { Remarks: Cut about } 45^{\circ} \text { to growth direction }\end{array}$ \\
\end{tabular}


Table S2. Stable-isotope data of the studied ice wedges incl. samples excluded from further analysis.

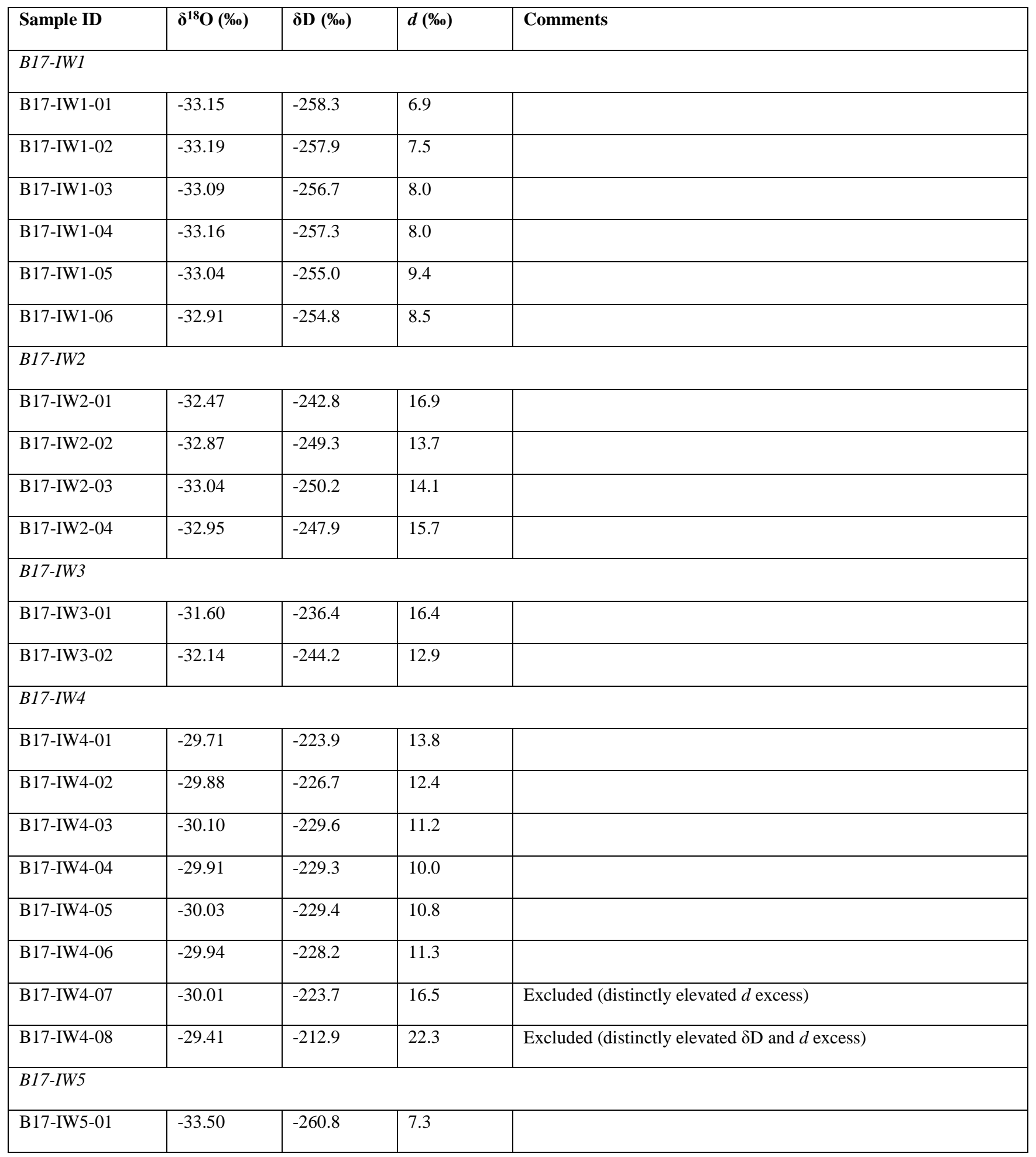




\begin{tabular}{|c|c|c|c|c|}
\hline B17-IW5-02 & -34.37 & -267.5 & 7.4 & \\
\hline B17-IW5-03 & -34.52 & -269.4 & 6.8 & \\
\hline B17-IW5-04 & -34.67 & -269.7 & 7.7 & \\
\hline B17-IW5-05 & -34.21 & -265.6 & 8.1 & \\
\hline B17-IW5-06 & -34.04 & -264.6 & 7.7 & \\
\hline B17-IW5-07 & -34.63 & -268.8 & 8.2 & \\
\hline B17-IW5-08 & -35.43 & -274.9 & 8.5 & \\
\hline B17-IW5-09 & -35.97 & -279.0 & 8.8 & \\
\hline B17-IW5-10 & -36.17 & -280.6 & 8.7 & \\
\hline B17-IW5-11 & -36.06 & -280.3 & 8.2 & \\
\hline B17-IW5-12 & -35.07 & -270.2 & 10.3 & \\
\hline \multicolumn{5}{|l|}{ B17-IW6 } \\
\hline B17-IW6-01 & -33.91 & -251.4 & 19.9 & Excluded (distinctly elevated $\delta \mathrm{D}$ and $d$ excess) \\
\hline B17-IW6-02 & -35.52 & -274.3 & 9.8 & \\
\hline B17-IW6-03 & -35.43 & -274.9 & 8.5 & \\
\hline B17-IW6-04 & -34.99 & -268.8 & 11.1 & \\
\hline B17-IW6-05 & -34.27 & -259.7 & 14.4 & \\
\hline B17-IW6-06 & -33.19 & -246.3 & 19.2 & Excluded (distinctly elevated $\delta \mathrm{D}$ and $d$ excess) \\
\hline A17-IW3-01 & -25.73 & -200.1 & 5.7 & Excluded (high sediment content, elevated $\delta^{18} \mathrm{O}$ and $\delta \mathrm{D}$ ) \\
\hline A17-IW3-02 & -28.15 & -220.5 & 4.7 & \\
\hline A17-IW3-03 & -28.42 & -221.8 & 5.6 & \\
\hline A17-IW3-04 & -28.84 & -225.7 & 5.1 & \\
\hline A17-IW3-05 & -29.15 & -228.0 & 5.2 & \\
\hline A17-IW3-06 & -29.62 & -230.9 & 6.0 & \\
\hline A17-IW3-07 & -30.40 & -236.6 & 6.6 & \\
\hline A17-IW3-08 & -29.79 & -231.1 & 7.3 & \\
\hline A17-IW3-09 & -28.63 & -223.9 & 5.1 & \\
\hline
\end{tabular}




\begin{tabular}{|l|l|l|l|l|}
\hline A17-IW3-10 & -28.26 & -221.7 & 4.4 & \\
\hline A17-IW3-11 & -28.56 & -223.1 & 5.5 & \\
\hline
\end{tabular}


Table S3. Stable isotope data of Marine Isotope Stage 3 ice wedges in northeast Siberia showing mean values of $\delta^{18} \mathrm{O}$. $\delta \mathrm{D}$ and $d$ and respective standard deviations.

\begin{tabular}{|c|c|c|c|c|c|c|c|c|c|c|}
\hline $\begin{array}{l}\text { Site } \\
\text { ID. }\end{array}$ & Site & ${ }^{\circ} \mathbf{N}$ & ${ }^{\circ} \mathbf{E}$ & $\begin{array}{l}\text { Age } \\
{\left[\mathrm{ka}^{14} \mathrm{C} \text { BP }\right]}\end{array}$ & $\begin{array}{l}\delta^{18} \mathrm{O} \\
{[\% 0]}\end{array}$ & $\begin{array}{l}\delta \mathrm{D} \\
{[\% \circ]}\end{array}$ & $\begin{array}{l}d \\
{[\% \circ]}\end{array}$ & $\mathbf{N}$ & Reference & profile ID \\
\hline 1 & $\begin{array}{l}\text { Western Laptev Sea. } \\
\text { Mamontov Klyk }\end{array}$ & 73.6 & 117.1 & 27.2 & $-30.5 \pm 0.7$ & $-241.5 \pm 6.2$ & $2.2 \pm 0.6$ & 4 & Magens (2005) & MAK-IW-18 \\
\hline 2 & Lena Delta. Nagym & 72.9 & 123.2 & $>54.5$ to 44.2 & $-29.3 \pm 0.3$ & $-228.2 \pm 3.1$ & $5.8 \pm 0.9$ & 12 & $\begin{array}{l}\text { Schirrmeister et } \\
\text { al. (2003) }\end{array}$ & Nag6-20-I \\
\hline 3 & $\begin{array}{l}\text { Lena Delta. } \\
\text { Khardang }\end{array}$ & 73.0 & 124.2 & $>52.1$ to 28.1 & $-30.1 \pm 1.3$ & $-239.3 \pm 9.0$ & $1.6 \pm 2.2$ & 10 & $\begin{array}{l}\text { Schirrmeister et } \\
\text { al. (2011) }\end{array}$ & Kha-3-I \\
\hline 4 & $\begin{array}{l}\text { Lena Delta. } \\
\text { Kurungnakh }\end{array}$ & 72.3 & 126.3 & 50.0 to 31.9 & $-31.6 \pm 0.6$ & $-247.6 \pm 4.0$ & $5.3 \pm 1.2$ & 11 & $\begin{array}{l}\text { Schirrmeister et } \\
\text { al. } \\
\text { Wetterich et al. } \\
(2003) \text {; }\end{array}$ & Bkh3-I-3. Bkh IW II \\
\hline 5 & $\begin{array}{l}\text { Lena Delta. Sobo } \\
\text { Sise }\end{array}$ & 72.5 & 128.3 & 47.7 to 26.9 & $-29.7 \pm 1.1$ & $-230.7 \pm 8.7$ & $7.2 \pm 0.9$ & 16 & $\begin{array}{l}\text { Opel } \\
\text { (unpublished) }\end{array}$ & SOB14-IW3 \\
\hline 6 & $\begin{array}{l}\text { Central Laptev Sea. } \\
\text { Bykovsky }\end{array}$ & 72.0 & 129.3 & 50 to 30 & $-30.8 \pm 1.6$ & $-242.8 \pm 11.4$ & $3.7 \pm 1.7$ & 145 & $\begin{array}{l}\text { Meyer et al. } \\
\text { (2002a). } \\
\text { Meyer/Opel } \\
\text { (unpublished) }\end{array}$ & $\begin{array}{l}\text { MKh 3 (2 horizontal } \\
\text { transects. } 1 \text { vertical } \\
\text { transect). LD10-18 }\end{array}$ \\
\hline 7 & $\begin{array}{l}\text { Central Laptev Sea. } \\
\text { Muostakh }\end{array}$ & 71.6 & 130.0 & 46.8 to 28.3 & $-31.9 \pm 1.2$ & $-250.4 \pm 9.4$ & $5.1 \pm 0.9$ & 85 & $\begin{array}{l}\text { Meyer/Opel } \\
\text { (unpublished) }\end{array}$ & $\begin{array}{l}\text { MUO12-IW7. } \\
\text { MUO12-IW8 }\end{array}$ \\
\hline 8 & $\begin{array}{l}\text { Central Laptev Sea. } \\
\text { Buor Khaya }\end{array}$ & 71.6 & 132.2 & $>48.0$ to 30.1 & $-31.2 \pm 1.0$ & $-245.0 \pm 7.8$ & $4.5 \pm 0.7$ & 26 & $\begin{array}{l}\text { Schirrmeister et } \\
\text { al. (2017) }\end{array}$ & Buo-04. BK8 \\
\hline 9 & $\begin{array}{l}\text { New Siberian } \\
\text { Islands. Belkovsky }\end{array}$ & 75.4 & 135.6 & 47.7 to $>39.8$ & $-31.3 \pm 0.4$ & $-243.2 \pm 4.6$ & $7.0 \pm 2.2$ & 21 & $\begin{array}{l}\text { Schirrmeister/ } \\
\text { Meyer } \\
\text { (unpublished) }\end{array}$ & BEL-IW-1 \\
\hline 10 & $\begin{array}{l}\text { New Siberian } \\
\text { Islands. Stolbovoy }\end{array}$ & 74.1 & 136.1 & $>50.0$ to $>40.8$ & $-31.7 \pm 0.4$ & $-248.0 \pm 4.9$ & $5.3 \pm 1.9$ & 16 & $\begin{array}{l}\text { Schirrmeister/ } \\
\text { Meyer } \\
\text { (unpublished) }\end{array}$ & STO-IW-1 \\
\hline
\end{tabular}




\begin{tabular}{|c|c|c|c|c|c|c|c|c|c|c|}
\hline 11 & $\begin{array}{l}\text { New Siberian } \\
\text { Islands. Kotel'ny }\end{array}$ & 74.7 & 138.5 & 52.8 to 35.4 & $-29.8 \pm 1.0$ & $-232.4 \pm 9.7$ & $5.6 \pm 2.5$ & 17 & $\begin{array}{l}\text { Schirrmeister/ } \\
\text { Meyer } \\
\text { (unpublished) }\end{array}$ & KYS-IW-1 \\
\hline 12 & $\begin{array}{l}\text { New Siberian } \\
\text { Islands. Bol'shoy } \\
\text { Lyakhovsky }\end{array}$ & 73.3 & 141.5 & 53.8 to 29.0 & $-31.0 \pm 1.2$ & $-240.9 \pm 11.0$ & $6.8 \pm 2.4$ & 196 & $\begin{array}{l}\text { Wetterich et al. } \\
(2014) \text {; Meyer } \\
\text { et al. (2002b); } \\
\text { Opel et al. } \\
(2017)\end{array}$ & $\begin{array}{l}\text { LYA-R9-1. LYA-R8- } \\
\text { 1. TZ-2-5. TZ-2-4. TZ- } \\
\text { 2-2. R10-1. R10-2. TZ- } \\
\text { 3-1. L7-18 }\end{array}$ \\
\hline 13 & $\begin{array}{l}\text { Dmitry Laptev Strait. } \\
\text { Oyogos Yar }\end{array}$ & 72.7 & 143.5 & 48.5 to 32.2 & $-30.8 \pm 1.3$ & $-240.8 \pm 10.9$ & $5.9 \pm 1.7$ & 150 & $\begin{array}{lll}\text { Opel et al. } \\
(2017) & & \end{array}$ & $\begin{array}{lr}\text { Oy7-01-4. } & \text { Oya-IW6. } \\
\text { Oya-IW2. } & \text { Oy7-06- } \\
\text { IW1. Oya-IW-5. } & \text { Oy7- } \\
\text { 06-IW2. Oy7-08 } & \text { IW1. } \\
\text { Oy7-08-IW3. } & \text { Oya- } \\
\text { IW3. Oya-IW4 }\end{array}$ \\
\hline 14 & $\begin{array}{l}\text { New Siberian } \\
\text { Islands. Novaya Sibir }\end{array}$ & 75.1 & 146.7 & $>51.7$ to $>38.3$ & $-33.5 \pm 0.6$ & $-260.7 \pm 6.2$ & $7.0 \pm 1.4$ & 5 & $\begin{array}{l}\text { Schirrmeister/ } \\
\text { Meyer } \\
\text { (unpublished) }\end{array}$ & NSI-IW-4 \\
\hline 15 & $\begin{array}{l}\text { Lower Kolyma. } \\
\text { Duvanny Yar }\end{array}$ & 68.6 & 159.1 & $>46.7$ to 32.2 & $-32.6 \pm 1.00$ & $-256.9 \pm 9.9$ & $3.7 \pm 2.0$ & 9 & Strauss (2010) & DY-01. DY-05 \\
\hline 16 & $\begin{array}{l}\text { Yana Highlands. } \\
\text { Batagay }\end{array}$ & 67.6 & 134.8 & 47.6 to 24.9 & $-34.9 \pm 0.9$ & $-271.0 \pm 6.4$ & $8.1 \pm 0.9$ & 12 & this study & B17-IW5 \\
\hline 16 & $\begin{array}{l}\text { Yana Highlands. } \\
\text { Batagay }\end{array}$ & 67.6 & 134.8 & undated & -35.7 & -276.0 & 9.2 & 59 & $\begin{array}{l}\text { Vasil'chuk et al. } \\
(2017)\end{array}$ & IW no. 2 \\
\hline 17 & $\begin{array}{l}\text { Central Yakutia } \\
\text { Aldan River. Tanda }\end{array}$ & 63.3 & 131.7 & undated & $-30.5 \pm 0.3$ & $-234.4 \pm 2.6$ & $9.5 \pm 0.5$ & 10 & $\begin{array}{l}\text { Schirrmeister } \\
\text { (unpublished) }\end{array}$ & Tan-I \\
\hline 18 & $\begin{array}{l}\text { Central Yakutia. } \\
\text { Aldan River. } \\
\text { Mamontova Gora }\end{array}$ & 63.0 & 134.0 & 46.7 to 34.0 & $-30.5 \pm 0.6$ & $-236.6 \pm 4.8$ & $7.6 \pm 0.6$ & 18 & $\begin{array}{lll}\text { Popp et al. } \\
(2006)\end{array}$ & MGO-I \\
\hline
\end{tabular}




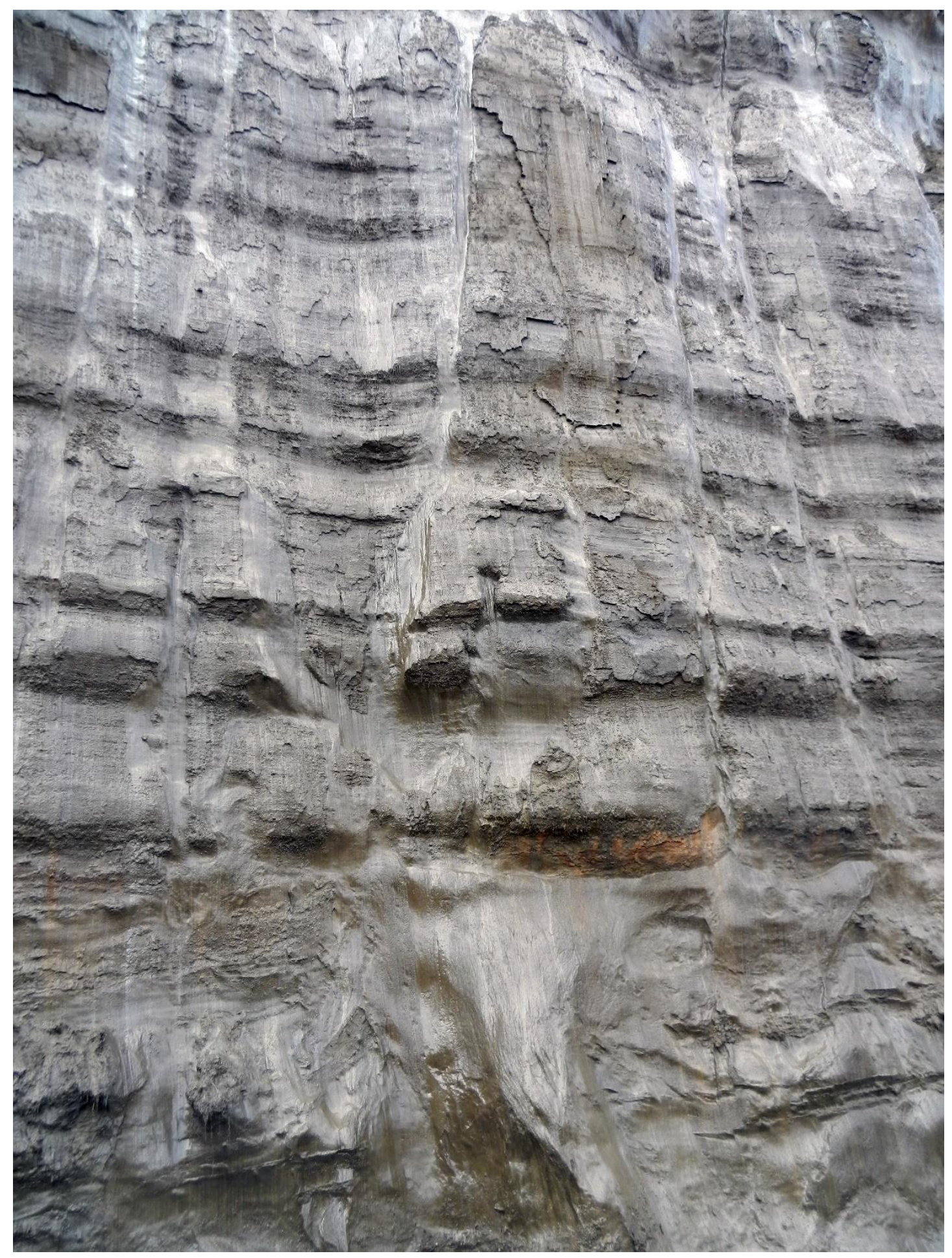

Figure S1. Erosional contact between Lower Ice Complex and Lower Sand. 


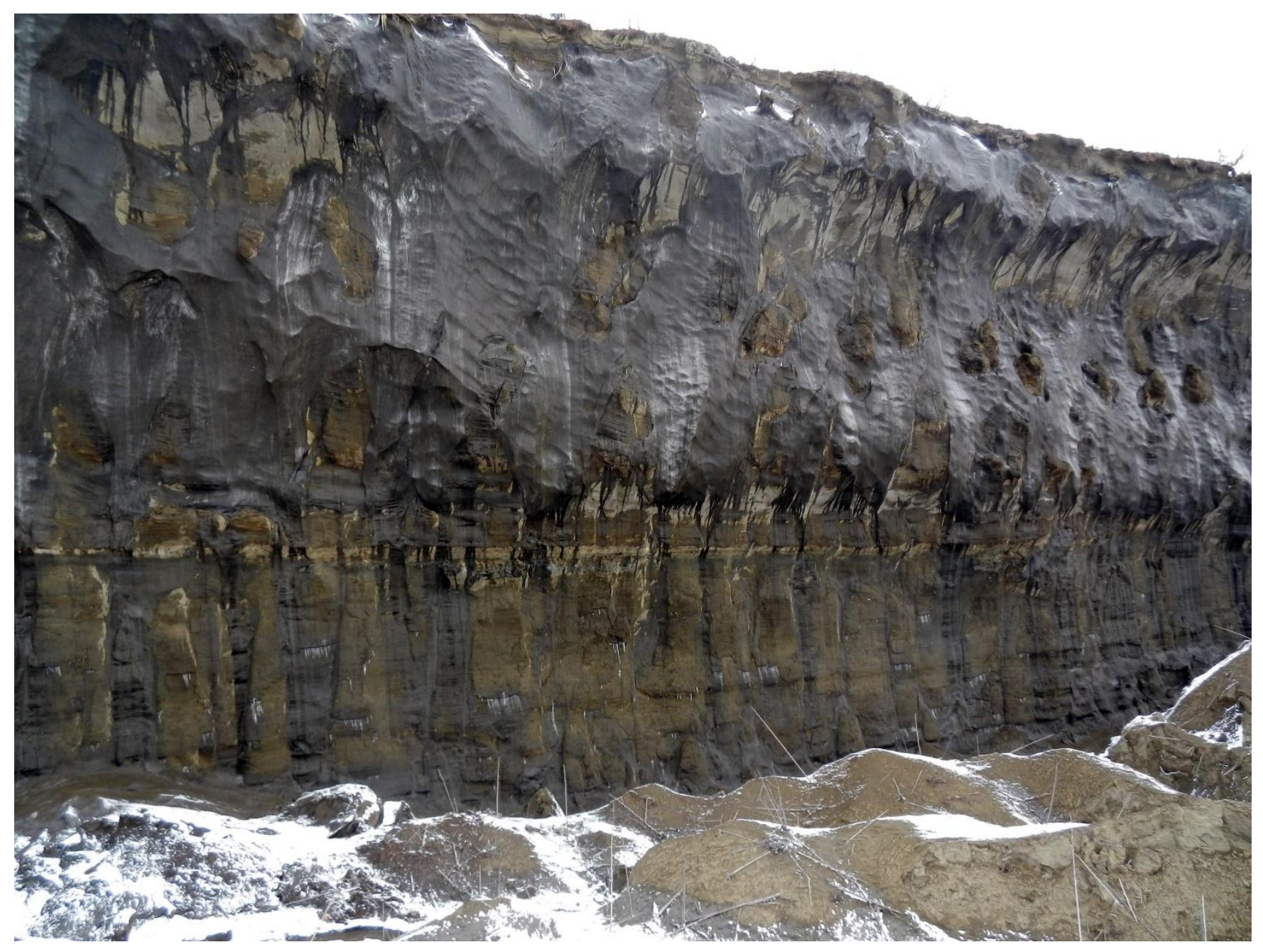

Figure S2. Transition of Lower Sand unit to Upper Ice Complex showing different kinds of ice-wedge toes. 

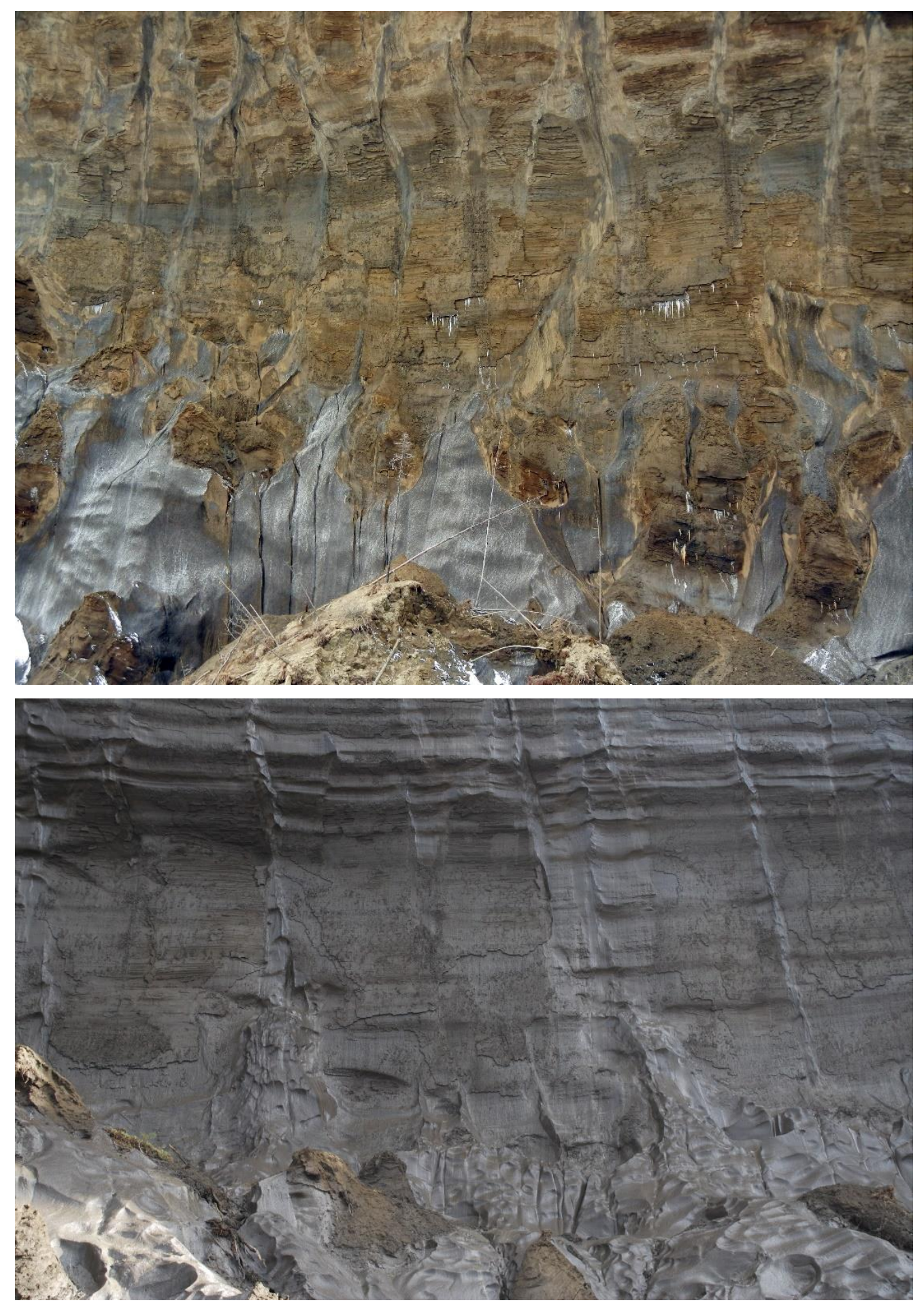

Figure S3. Transition from Upper Ice Complex to Upper Sand showing the upward tapering of the wide syngenetic ice wedges. the subvertical and downslope orientation of the narrow syngenetic composite wedges. as well as complexity of wedge shoulders. thaw unconformities and narrowing ice wedges. 


\section{References}

Magens. D.: Late Quaternary climate and environmental history of the Siberian Arctic - Permafrost Records from Cape Mamontovy Klyk. Laptev Sea. Diplom. Christian-Albrechts-Universität zu Kiel. 106 pp.. 2005.

Meyer. H.. Dereviagin. A. Y.. Siegert. C.. and Hubberten. H.-W.: Paleoclimate studies on Bykovsky Peninsula. North Siberia-

5 hydrogen and oxygen isotopes in ground ice. Polarforschung. 70. 37-51.2002a.

Meyer. H.. Dereviagin. A. Y.. Siegert. C.. Schirrmeister. L.. and Hubberten. H. W.: Palaeoclimate reconstruction on Big Lyakhovsky Island. North Siberia - Hydrogen and oxygen isotopes in ice wedges. Permafrost Periglacial Process.. 13. 91-105. 10.1002/ppp.416. 2002b.

Opel. T.. Wetterich. S.. Meyer. H.. Dereviagin. A. Y.. Fuchs. M. C.. and Schirrmeister. L.: Ground-ice stable isotopes and 10 cryostratigraphy reflect late Quaternary palaeoclimate in the Northeast Siberian Arctic (Oyogos Yar coast. Dmitry Laptev Strait). Climate of the Past. 13. 587-611. 10.5194/cp-13-587-2017. 2017.

Popp. S.. Diekmann. B.. Meyer. H.. Siegert. C.. Syromyatnikov. I.. and Hubberten. H. W.: Palaeoclimate signals as inferred from stable-isotope composition of ground ice in the Verkhoyansk foreland. Central Yakutia. Permafrost Periglacial Process. 17. 119-132. 10.1002/ppp.556. 2006.

15 Schirrmeister. L.. Grosse. G.. Schwamborn. G.. Andreev. A. A.. Meyer. H.. Kunitsky. V. V.. Kuznetsova. T. V.. Dorozhkina. M. V.. Pavlova. E. Y.. Bobrov. A. A.. and Oezen. D.: Late Quaternary History of the Accumulation Plain North of the Chekanovsky Ridge (Lena Delta. Russia): A Multidisciplinary Approach. Polar Geography. 27. 277-319. 10.1080/789610225. 2003.

Schirrmeister. L.. Grosse. G.. Schnelle. M.. Fuchs. M.. Krbetschek. M.. Ulrich. M.. Kunitsky. V.. Grigoriev. M.. Andreev. A..

20 Kienast. F.. Meyer. H.. Babiy. O.. Klimova. I.. Bobrov. A.. Wetterich. S.. and Schwamborn. G.: Late Quaternary paleoenvironmental records from the western Lena Delta. Arctic Siberia. Paleogeogr. Paleoclimatol. Paleoecol.. 299. 175-196. 10.1016/j.palaeo.2010.10.045. 2011.

Schirrmeister. L.. Schwamborn. G.. Overduin. P. P.. Strauss. J.. Fuchs. M. C.. Grigoriev. M.. Yakshina. I.. Rethemeyer. J.. Dietze. E.. and Wetterich. S.: Yedoma Ice Complex of the Buor Khaya Peninsula (southern Laptev Sea). Biogeosciences. 14.

25 1261-1283. 10.5194/bg-14-1261-2017. 2017.

Strauss. J.: Late Quaternary environmental dynamics at the Duvanny Yar key section. Lower Kolyma. East Siberia. Diplom. Universität Potsdam. 108 pp.. 2010.

Vasil'chuk. Y. K.. Vasil'chuk. J. Y.. Budantseva. N. A.. Vasil'chuk. A. K.. and Trishin. A. Y.: Isotope-geochemical characteristics of the Batagay Yedoma (Preliminary results). Arktika i Antarktika (Arctic and Antarctica). 1. 69-98. 2017.

30 Wetterich. S.. Kuzmina. S.. Andreev. A. A.. Kienast. F.. Meyer. H.. Schirrmeister. L.. Kuznetsova. T.. and Sierralta. M.: Palaeoenvironmental dynamics inferred from late Quaternary permafrost deposits on Kurungnakh Island. Lena Delta. Northeast Siberia. Russia. Quaternary Science Reviews. 27. 1523-1540. 10.1016/j.quascirev.2008.04.007. 2008.

Wetterich. S.. Tumskoy. V.. Rudaya. N.. Andreev. A. A.. Opel. T.. Meyer. H.. Schirrmeister. L.. and Huls. M.: Ice Complex formation in arctic East Siberia during the MIS3 Interstadial. Quaternary Science Reviews. 84. 39-55. 10.1016/j.quascirev.2013.11.009. 2014. 